\title{
Nanomagnetic Particles for SQUID-Based Magnetically Labeled Immunoassay
}

\author{
H. E. Horng, S. Y. Yang, Y. W. Huang, W. Q. Jiang, C.-Y. Hong, and H. C. Yang
}

\begin{abstract}
With the increasing importance of SQUID-based magnetically labeled immunoassay, the study on the synthesis of controllable sizes of magnetic nanoparticles plays a role to promote the accuracy of the immunoassay. In this work, $\mathrm{Fe}_{3} \mathrm{O}_{4}$ nano-particles coated with a suitable bio-probe (biotin) are synthesized through chemical co-precipitation process to probe the bio-target (avidin). Through the synthesis developed here, the particle hydrodynamic diameter can be adjusted from 30 to 90 nm, which provide candidates for probing various bio-targets in the future. The amount of the magnetically labeled avidin is then analyzed via measuring the saturated magnetization or the remanence of the sample by using a SQUID magnetometer.
\end{abstract}

Index Terms-Immunoassay, nanomagnetic particles, SQUIDs.

\section{INTRODUCTION}

W ITH the rapid progress of nano-biotechnology, lots of new research topics have been created and have been attracting a great deal of interest from scientists and engineers. In this area, the exploration of nano-bio-diagnosis on antibody, antigen, cellular and/or genetic levels is both important and urgent because of the unique merits, like ultrahigh sensitivity. The popularly used types of bio-diagnosis, such as enzyme linked immunosorbent assay (ELISA) or fluorescence immunoassay (FIA), have disadvantages; for example, complicated processes or higher uncertainty in the accuracy due to the self-absorption/emission of detected bio-samples. To promote bio-diagnosis, numerous novel methods have been proposed and actively developed [1]-[5]. A promising candidate is the magnetically labeled bio-diagnosis because of its high efficiency, high convenience, high accuracy and versatile functions [6]-[8].

For magnetically labeled bio-diagnosis (MLD), the bio-probe (say biotin) is coated onto the surface of magnetic nano-particles, which are dispersed in water. The coated nano-particles in the magnetic fluid associate with the target bio-molecular (avidin) to form magnetic clusters. Then, the amount of the

Manuscript received October 5, 2004. This work was supported by the NSC under Grants NSC93-2120-M-003-001, 93-2112-M-003-017, and 93-2112-M002-041, and by MOE under Grant 91-N-FA01-2-4-2.

H. E. Horng is with the Institute of Electro-optical Science and Technology, National Taiwan Normal University, Taipei 116, Taiwan and also with the Department of Physics, National Taiwan Normal University, Taipei 116, Taiwan (e-mail: phyfv001@scc.ntnu.edu.tw).

S. Y. Yang and Y. W. Huang are with the Institute of Electro-optical Science and Technology, National Taiwan Normal University, Taipei 116, Taiwan.

W. Q. Jiang is with the Department of Physics, National Taiwan University, Taipei 106, Taiwan and also with the Department of Chemistry, University of Science and Technology of China, Hefei, 230026, P. R. China.

C.-Y. Hong is with the Department of Mechanical and Automation Engineering, Da-Yeh University, Chang-Hwa 515, Taiwan.

H. C. Yang is with the Department of Physics, National Taiwan University, Taipei 106, Taiwan.

Digital Object Identifier 10.1109/TASC.2005.849995 target bio-molecule is detected by measuring the magnetic signals of the magnetic clusters [9]-[11]. At present, the magnetic properties such as magnetic relaxation, magnetic remanence, and ac magnetic susceptibility of these magnetic clusters are detected. To achieve a very high sensitivity to the magnetic characterization of the MLD system, the superconducting quantum interference devices (SQUID) is adapted as a sensor because the SQUID is the most sensitive device to measure magnetic moments in the world. Therefore, SQUID-based magnetically labeled diagnosis systems are the most promising candidate for MLD.

It was pointed out that the resolution and the accuracy for MLD greatly depend on the mean value and the dispersion of the nano-particle size. For example, magnetic particles with larger diameters $(\sim 20 \mathrm{~nm})$ are used for the relaxation measurement in order to distinguish the Brownian relaxation time constant of a single particle from the Néel relaxation time constant [10]. For the ac susceptibility measurement, a higher uniformity in nanoparticle size is required. Hence, the study on synthesis methods to prepare highly homogeneous magnetic nanoparticles of controlled sizes is an important issue in developing MLD. In this work, we study the preparation of bio-compatible magnetic nano-particles for SQUID-based immunoassay.

\section{EXPERIMENTAL DETAILS}

A flow chart to illustrate the synthesis of the bio-compatible magnetic $\mathrm{Fe}_{3} \mathrm{O}_{4}$ nanoparticles is shown in Fig. 1 [12]. 50-ml 0.20-M ferrite solution containing stoichiometric ratio 1:2 ferrous sulfate hepta-hydrate $\left(\mathrm{FeSO}_{4} \cdot 7 \mathrm{H}_{2} \mathrm{O}\right)$ and ferric chloride hexa-hydrate $\left(\mathrm{FeCl}_{3} \cdot 6 \mathrm{H}_{2} \mathrm{O}\right)$ was well mixed with an equal volume of aqueous dextran. The dextran (molecular weight $=74000$ ) was selected as a surfactant of $\mathrm{Fe}_{3} \mathrm{O}_{4}$ particles dispersed in water by taking the hydrophilic, biocompatibility, toxicity, and easy preparation into account for biomedical applications. Then appropriate amount of urea $\mathrm{CO}\left(\mathrm{NH}_{2}\right)_{2}$ was added into the mixed solution. The mixture was heated gradually up to $90{ }^{\circ} \mathrm{C}$ to decompose urea. The amount of decomposed urea was adjusted by controlling the heating duration after the temperature reached $90{ }^{\circ} \mathrm{C}$. After that, while stirring rigorously, the mixture was titrated to raise the $\mathrm{pH}$ to around $10-11$ by the addition drop wise of $3.5-\mathrm{M}$ ammonium hydroxide at room temperature. The black $\mathrm{Fe}_{3} \mathrm{O}_{4}$ particles were synthesized in this step. Aggregates and excess unbound dextran were removed by centrifugation and gel filtration chromatography. The stable, purified water-based magnetic fluid containing dextran-coated $\mathrm{Fe}_{3} \mathrm{O}_{4}$ nano-particles was obtained with a concentration of about $10 \mathrm{mg} \mathrm{Fe} / \mathrm{ml}$. Then, 


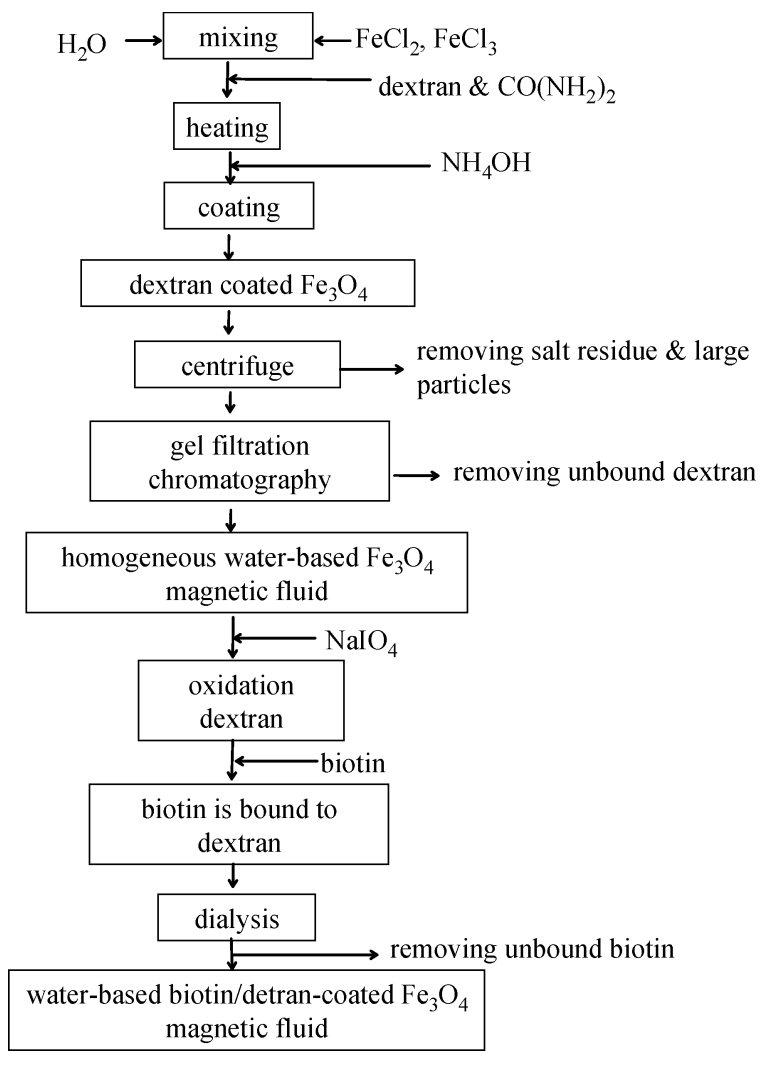

Fig. 1. Flow chart for preparation of bio-compatible $\mathrm{Fe}_{3} \mathrm{O}_{4}$ magnetic nanoparticles dispersed in water.

biotin was bound onto the dextran on the $\mathrm{Fe}_{3} \mathrm{O}_{4}$ particles through the oxidation of dextran by adding $\mathrm{NaIO}_{4}$. Thus, biotin-dextran-coated $\mathrm{Fe}_{3} \mathrm{O}_{4}$ particles dispersed in water were obtained for use in detecting avidin. Finally, the unbound biotin was removed by dialyzing the biotin-dextran coated $\mathrm{Fe}_{3} \mathrm{O}_{4}$ nano-particle solution. The crystalline of magnetic particles was analyzed using powder $x$-ray diffraction (XRD) in a Siemens D-500N diffractometer with a wavelength of $0.15418 \mathrm{~nm}$. The size distribution of $\mathrm{Fe}_{3} \mathrm{O}_{4}$ particles in water was evaluated by using laser scattering analysis of Microtrac Nanotrac-150.

\section{RESULTS AND DISCUSSION}

According to the synthesis process shown in Fig. 1, the $\mathrm{Fe}^{3+}$ in $\mathrm{FeCl}_{3} \cdot 6 \mathrm{H}_{2} \mathrm{O}$ reacts with the $\mathrm{Fe}^{2+}$ in $\mathrm{FeSO}_{4} \cdot 7 \mathrm{H}_{2} \mathrm{O}$ via

$$
2 \mathrm{Fe}^{3+}+\mathrm{Fe}^{2+}+8 \mathrm{OH}^{-} \rightarrow \mathrm{Fe}_{3} \mathrm{O}_{4}+\mathrm{H}_{2} \mathrm{O}
$$

to form $\mathrm{Fe}_{3} \mathrm{O}_{4}$ particles. The $\mathrm{OH}^{-}$in (1) is provided by decomposing urea at $90{ }^{\circ} \mathrm{C}$ via

$$
\begin{aligned}
\mathrm{CO}\left(\mathrm{NH}_{2}\right)_{2}+\mathrm{H}_{2} \mathrm{O} & \rightarrow 2 \mathrm{NH}_{3}+\mathrm{CO}_{2} \\
\mathrm{NH}_{3} \cdot \mathrm{H}_{2} \mathrm{O} & \rightarrow \mathrm{NH}_{4}^{+}+\mathrm{OH}^{-}
\end{aligned}
$$

Since the concentration of $\mathrm{OH}^{-}$released from urea is not high enough to activate the $\mathrm{Fe}_{3} \mathrm{O}_{4}$ formation, ammonia solution is required to be titrated into the mixture. After the formation of $\mathrm{Fe}_{3} \mathrm{O}_{4}$ particles, dextran is attached onto the particles. The dextran acts not only a surfactant between the particles and water, but also serves as a connection between the bio-probe (biotin) and the magnetic particles.

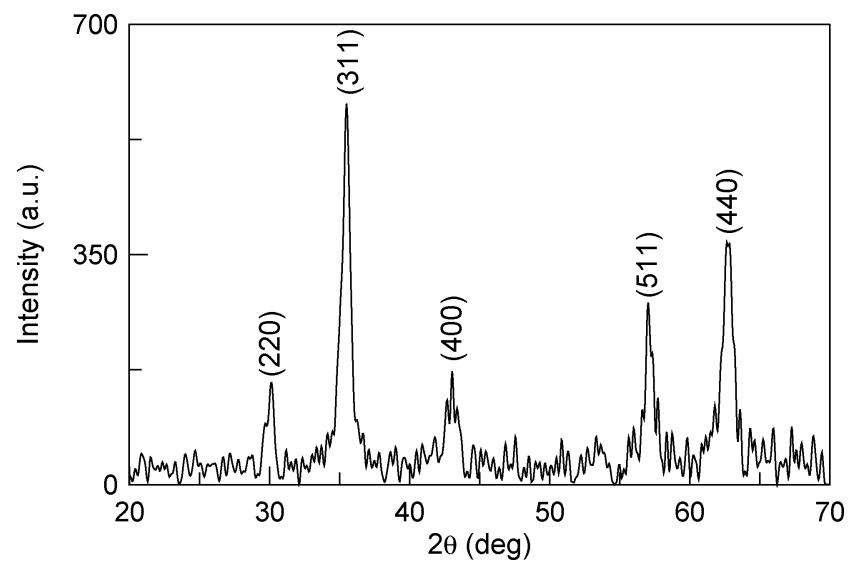

Fig. 2. $\theta-2 \theta$ x-ray powder diffraction pattern of the synthesized magnetic particles. The indexes are for the $\mathrm{Fe}_{3} \mathrm{O}_{4}$ phase.

In order to associate dextran with biotin, the dextran on the particles is oxidized with $\mathrm{NaIO}_{4}$ to change the hydroxyl group $(-\mathrm{COH})$ into an aldehyde group $(-\mathrm{CHO})$. Then, the aldehyde group $(-\mathrm{CHO})$ in dextran can react to the amino group $\left(-\mathrm{NH}_{2}\right)$ in biotin to achieve bio-probes for avidin on magnetic particles, as shown in

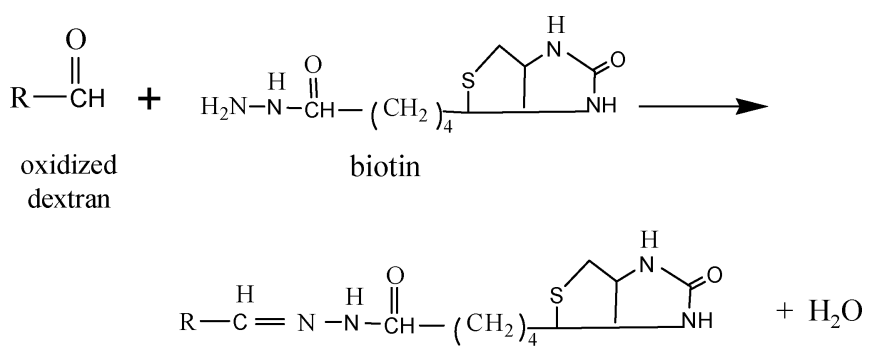

dextran-biotin association

where $\mathrm{R}$ in oxidized dextran sticks onto magnetic particles. Thus, we can obtain a homogeneous water-based biotin-dextran coated $\mathrm{Fe}_{3} \mathrm{O}_{4}$ magnetic fluid. Fig. 2 shows the XRD pattern for the synthesized magnetic particles. It is clear that the magnetic particles are single phase of $\mathrm{Fe}_{3} \mathrm{O}_{4}$.

Since the sensitivity of MLD heavily relies on the average diameter and its standard deviation of $\mathrm{Fe}_{3} \mathrm{O}_{4}$ particles, the size distribution of $\mathrm{Fe}_{3} \mathrm{O}_{4}$ particles was measured. Fig. 3(a) gives a typical size distribution of the isolated biotin-dextran coated $\mathrm{Fe}_{3} \mathrm{O}_{4}$ particles. The mean value of the hydrodynamic diameter of $\mathrm{Fe}_{3} \mathrm{O}_{4}$ particles is $30.9 \mathrm{~nm}$ with a standard deviation of $21 \%$. According to our studies, the average diameter of $\mathrm{Fe}_{3} \mathrm{O}_{4}$ nanoparticles can be adjusted from 25 to $90 \mathrm{~nm}$ by controlling the period of time for decomposing urea at $90{ }^{\circ} \mathrm{C}$, as shown in Fig. 3(b). The flexibility in tuning particle size provides opportunities to investigate the most suitable size of magnetic nanoparticles for various MLD systems.

After adding the bio-target avidin into the magnetic fluid, the biotin-dextran coated $\mathrm{Fe}_{3} \mathrm{O}_{4}$ particles were attached together to form magnetic clusters because that avidin was able to associate with biotins on different $\mathrm{Fe}_{3} \mathrm{O}_{4}$ particles. As a result, the mean value of the magnetic clusters in the solution becomes larger 

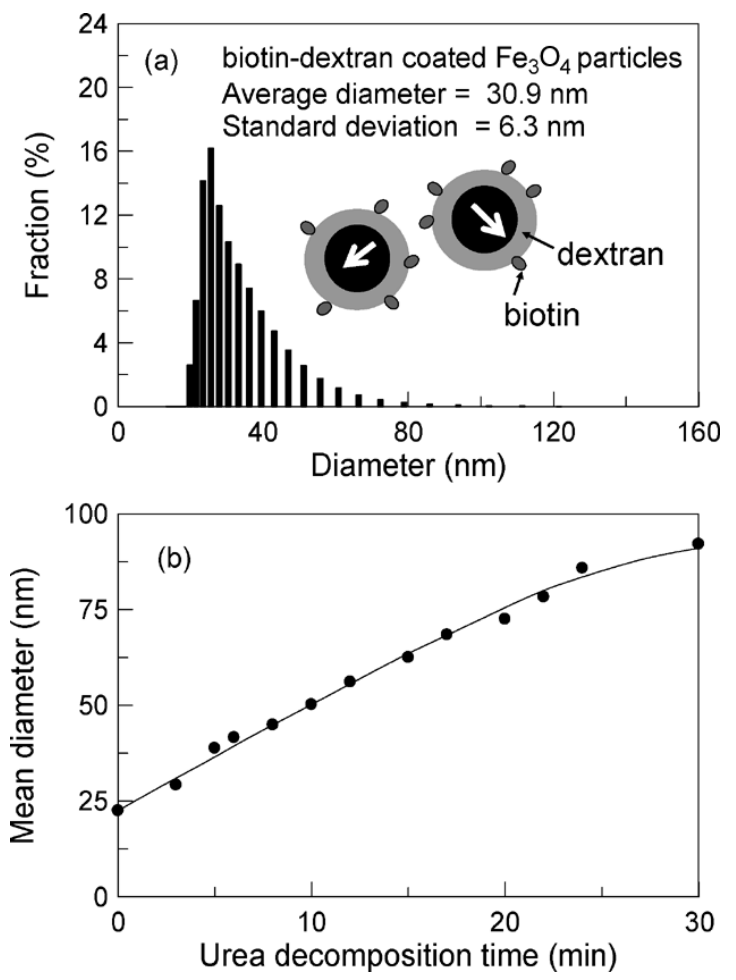

Fig. 3. (a) Size distribution of isolated biotin-dextran coated $\mathrm{Fe}_{3} \mathrm{O}_{4}$ nano-particles. (b) Mean diameter of $\mathrm{Fe}_{3} \mathrm{O}_{4}$ particles as functions of the decomposition time of urea. The inset in (a) illustrate two isolated biotin-dextran coated $\mathrm{Fe}_{3} \mathrm{O}_{4}$ nanoparticles. The arrows denote the magnetic dipole moments.

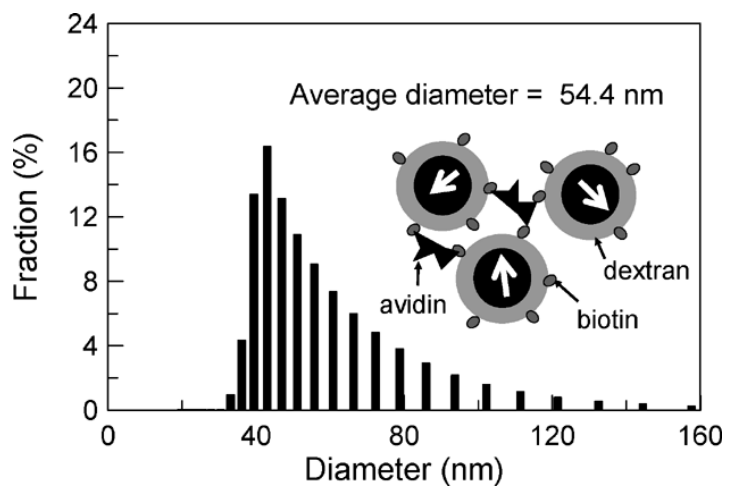

Fig. 4. Size distribution of $\mathrm{Fe}_{3} \mathrm{O}_{4}$ particles reacting with the detected bio-target avidin. The inset illustrates the magnetic clusters formed with avidin and biotin-dextran coated $\mathrm{Fe}_{3} \mathrm{O}_{4}$ particles.

as compared with that of isolated $\mathrm{Fe}_{3} \mathrm{O}_{4}$ particles. This is evidenced by the results shown in Fig. 4. It was found that the mean size increased from $30.9 \mathrm{~nm}$ for isolated particles to $54.4 \mathrm{~nm}$ for magnetic clusters when the biotin-dextran $\mathrm{Fe}_{3} \mathrm{O}_{4}$ particles reacted with avidin. It is noted that when various amounts of avidin are added to react with the biotin-dextran coated $\mathrm{Fe}_{3} \mathrm{O}_{4}$ particles, different values of the mean size of magnetic clusters are observed. Fig. 5(a) gives the experimental relationship between the mean size of magnetic clusters and the amount of the added avidin. The mean size of the magnetic clusters increases linearly with the increasing amount of avidin of 30 to $100 \mu \mathrm{g}$.

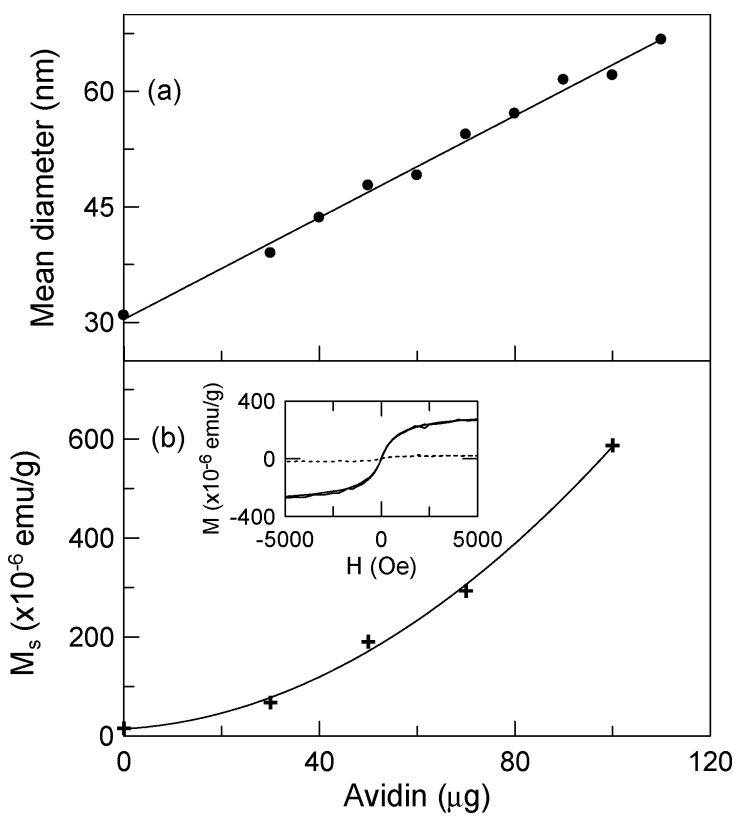

Fig. 5. Relationship between (a) the mean size of the magnetic clusters, (b) the saturated magnetization of the magnetic clusters and the amount of avidin. The inset in (b) shows the magnetic hysteresis curves for the filtrated biotin-dextran coated $\mathrm{Fe}_{3} \mathrm{O}_{4}$ with (solid line) or without (dashed line) binding to avidin.

In addition to the mean size of the magnetic clusters, the amount of the added avidin can be analyzed by detecting its magnetic properties, as is done in a magnetically labeled immunoassay. Here, the saturated magnetization and the magnetic remanence of the magnetic clusters were measured by using a SQUID magnetometer. To do this, the magnetic clusters have to be separated from isolated particles that do not react with avidin. This can be achieved by filtering the sample through a micro-filter with nano-sized holes of $50 \mathrm{~nm}$ in diameter. After drying the filtered sample, the magnetic hysteresis of the sample was measured. Since the mean size of the magnetic clusters is different for the samples added with various amounts of avidin, the amount of the residual magnetic clusters on the micro-filters is different, in turns, various values of saturated magnetization $\mathrm{M}_{\mathrm{s}}$ should be observed. Two typical magnetic hysteresis curves for the samples with (solid line)/without (dashed line) reacting to avidin are shown in the inset of Fig. 5(b). The sample with the association to avidin exhibits a significant nonzero value of saturated magnetization. Fig. 5(b) plots the saturated magnetization of the samples as a function of the amount of added avidin. It is clear that the more the avidin, the higher the saturated magnetization.

Besides exhibiting a higher saturated magnetization, the sample with magnetic clusters should exhibit a higher value of magnetic remanence $M_{R}$. The value of $M_{R}$ of each sample can be identified from its magnetic hysteresis curve taken by using a SQUID magnetometer. Fig. 6 shows $M_{R}$ of the magnetic clusters as a function of the amount of the added avidin. A linear relationship was observed. The results shown in Figs. 5 and 6 reveal that the synthesized biotin-dextran coated $\mathrm{Fe}_{3} \mathrm{O}_{4}$ magnetic nanoparticles can be used as a labeling indicator in MLD. 


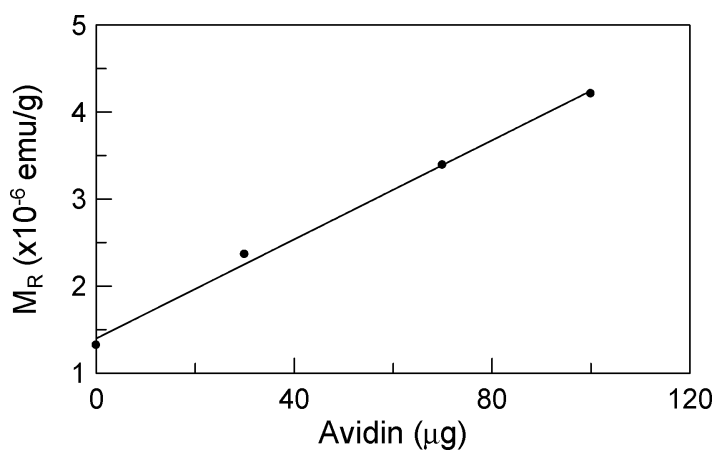

Fig. 6. Magnetic remanence of magnetic clusters as a function of amount of avidin.

\section{CONCLUSION}

Biocompatible $\mathrm{Fe}_{3} \mathrm{O}_{4}$ magnetic nanoparticles have been synthesized. We have further demonstrated that the biocompatible $\mathrm{Fe}_{3} \mathrm{O}_{4}$ magnetic nanoparticles can be used for SQUID-based magnetically labeled detection for bio-molecules, such as avidin. The amount of detected bio-target can be probed quantitatively by measuring the saturated magnetization or the magnetic remanence by using a SQUID magnetometer.

\section{REFERENCES}

[1] J. K. Gimzewski and C. Joachim, "Nanoscale science of single molecules using local probes," Science, vol. 283, pp. 1683-1688, 1999.
[2] R. Matzke, K. Jacobson, and M. Radmacher, "Direct, high-resolution measurement of furrow stiffening during division of adherent cells," $\mathrm{Na}$ ture-Cell Bio., vol. 3, pp. 607-610, 2001.

[3] R. J. Linhardt and T. Toida, "CAPILLARY ELECTROPHORESIS: ultrahigh resolution separation comes of age," Science, vol. 298, pp. 1441-1442, 2002.

[4] J. T. Groves and M. L. Dustin, "Supported planar bilayers in studies on immune cell adhesion and communication," J. Immunological Methods, vol. 278, pp. 19-32, 2003.

[5] P. Alivisatos, "The use of nanocrystals in biological detection," NatureBiotech., vol. 22, pp. 47-52, 2004.

[6] S. Katsura, T. Yasuda, K. Hirano, A. Mizuno, and S. Tanaka, "Development of a new detection method for DNS molecules," Supercond. Sci. Technol., vol. 14, pp. 1131-1134, 2001.

[7] S. K. Lee, W. R. Myers, H. L. Grossman, H.-M. Cho, Y. R. Chemla, and J. Clarke, "Magnetic gradiometer based on a high-transition temperature superconducting quantum interference device for improved sensitivity of a biosensor," Appl. Phy. Lett., vol. 81, pp. 3094-3096, 2002.

[8] K. Enpuku, T. Minotani, T. Gima, Y. Kuroki, Y. Itoh, M. Yamashita, Y. Katakura, and S. Kuhara, "Detection of magnetic nanoparticles with superconducting quantum interference device (SQUID) magnetometer and application to immunoassays," Jpn. J. Appl. Phys., vol. 38, pp. L1102-L1105, 1999.

[9] J. Bauer, "Advances in cell separation: recent developments in counterflow centrifugal elutriation and continuous flow cell separation," $J$ Chromatography B: Biomedical Sci. Appl., vol. 722, pp. 55-69, 1999.

[10] R. Kötitz, W. Weitschies, L. Trahms, W. Brewer, and W. Semmler, "Determination of the binding reaction between avidin and biotin by relaxation measurements of magnetic nanoparticles," J. Magn. Magn. Mater. vol. 194, pp. 62-68, 1999.

[11] P. J. Fisher, M. J. Springett, A. B. Dietz, P. A. Bulur, and S. Vuk-Pavlovic, "Immunomagnetic separation reagents as markers in electron microscopy," J. Immunological Methods, vol. 262, pp. 95-101, 2002.

[12] W. Q. Jiang, H. C. Yang, S. Y. Yang, H. E. Horng, J. C. Hung, Y. C. Chen, and C.-Y. Hong, "Preparation and properties of superparamagnetic nanoparticles with narrow size distribution and biocompatible," $J$. Magn. Mater, vol. 283, pp. 210-214, 2004. 